\title{
Loss of Heterozygosity at the Calcium Regulation Gene Locus on Chromosome 10q in Human Pancreatic Cancer
}

\author{
Jin Long*, Zhong-Bo Zhang, Zhe Liu, Yuan-Hong Xu, Chun-Lin Ge
}

\begin{abstract}
Background: Loss of heterozygosity (LOH) on chromosomal regions is crucial in tumor progression and this study aimed to identify genome-wide LOH in pancreatic cancer. Materials and Methods: Single-nucleotide polymorphism (SNP) profiling data GSE32682 of human pancreatic samples snap-frozen during surgery were downloaded from Gene Expression Omnibus database. Genotype console software was used to perform data processing. Candidate genes with LOH were screened based on the genotype calls, SNP loci of LOH and dbSNP database. Gene annotation was performed to identify the functions of candidate genes using NCBI (the National Center for Biotechnology Information) database, followed by Gene Ontology, INTERPRO, PFAM and SMART annotation and UCSC Genome Browser track to the unannotated genes using DAVID (the Database for Annotation, Visualization and Integration Discovery). Results: The candidate genes with LOH identified in this study were $M C U, M I C U 1$ and $O I T 3$ on chromosome 10. MCU was found to encode a calcium transporter and $M I C U 1$ could encode an essential regulator of mitochondrial $\mathrm{Ca}^{2+}$ uptake. OIT3 possibly correlated with calcium binding revealed by the annotation analyses and was regulated by a large number of transcription factors including $S T A T, S O X 9, C R E B, N F-k B, P P A R G$ and $p 53$. Conclusions: Global genomic analysis of SNPs identified $M I C U 1, M C U$ and $O I T 3$ with $\mathrm{LOH}$ on chromosome 10, implying involvement of these genes in progression of pancreatic cancer.
\end{abstract}

Keywords: pancreatic cancer - loss of heterozygosity - single-nucleotide polymorphism

Asian Pac J Cancer Prev, 16 (6), 2489-2493

\section{Introduction}

Loss of heterozygosity $(\mathrm{LOH})$ is a common genetic alteration in cancer genomes, which derives from heterozygous deletion of one of the two alleles, or duplication of a maternal or paternal chromosome or chromosomal region and concurrent loss of the other allele (Frampton and King, 2013). LOH on chromosomal regions containing key tumor suppressor genes has been considered as a significant contributor to drive cancer progression by inactivating the suppressor genes of tumor (Baker et al., 2009), including pancreatic cancer. For example, $p 53$ tumor suppressor gene is identified to experience $\mathrm{LOH}$ accompanied by mutation in pancreatic cancer cell lines (Butz et al., 2003). Cooperating with mutation and/or loss of $p 53$, the LOH of BRCAl and $B R C A 2$ may contribute to the progression of pancreatic cancer (Lucas et al., 2013). Besides, LOH in THBS2 (6q), pl6 (9p) and $A P C$ (5q) are also demonstrated to carry the worst prognosis for resected pancreatic ductal and ampullary adenocarcinomas (Franko et al., 2008). By using genome-wide $\mathrm{LOH}$ maps in pancreatic cancer, $\mathrm{LOH}$ is found with high frequency at various chromosomes such as 3p, 6p, 6q, 8q, 9p, 9q, 12q, 13q, 17p, 18q and 22q (Lin et al., 2008). However, candidate genes with $\mathrm{LOH}$ in pancreatic cancer remains rarely investigated.

Hybridization to single-nucleotide polymorphism (SNP) arrays is an efficient method to detect genome-wide cancer LOH by identifying the absence of heterozygous loci (Beroukhim et al., 2006; Staaf et al., 2008). Thus, the goal of this study was to further identify candidate genes with $\mathrm{LOH}$ in pancreatic cancer by performing a genomewide analysis of LOH using the SNP arrays deposited in public database by Donahue et al. (2012), followed by annotation analysis and transcription factors screening in an attempt to explain the involvement of these genes in pancreatic cancer.

\section{Materials and Methods}

\section{SNP expression profiling data}

The SNP expression profiling data GSE32682 (Donahue et al., 2012) of human pancreatic samples including 25 human pancreatic cancer and 7 nonmalignant pancreas samples snap-frozen during surgery were downloaded from the GEO (Gene Expression 
Jin Long et al

Omnibus) database that is developed as a repository of microarrays, chips, hybridization arrays and high throughput gene expression data. The gene expressions of these samples were investigated by Affymetrix GenomeWide Human SNP 6.0 Array (GPL6801).

Data processing and screening of candidate genes with $\mathrm{LOH}$

Affymetrix CEL files were analyzed using Genotype Console software (version 4.0; Affymetrix) for initial intensity quality control (QC) with the criterion of recommended contrast $\mathrm{QC}>0.4$, followed by generating SNP genotype calls using the Affymetrix Birdseed algorithm and filtrating SNPs loci with the excluded thresholds of no-call rate $\geq 10 \%$, minor allele frequency $<0.05$ and Hardy Weinberg Equilibrium (HWE) P-value 0.001 . Then, copy-neutral LOH $(\mathrm{CN}-\mathrm{LOH})$ analysis was performed with the threshold of MAPD $<0.04$ to identify the SNP loci of $\mathrm{LOH}$ that existed in over $50 \%$ of the pancreatic cancer samples while not in control samples. The candidate genes with $\mathrm{LOH}$ were screened based on the genotype calls, SNP loci of LOH and dbSNP database (Day, 2010).

\section{Gene annotation of Candidate genes}

The candidate genes with $\mathrm{LOH}$ were annotated by using Gene database in NCBI (the National Center for Biotechnology Information) and the unannotated genes were inputted into DAVID (the Database for Annotation, Visualization and Integration Discovery) for Gene Ontology (GO), INTERPRO, PFAM and SMART annotation (Sherman et al., 2007).

Transcription factors screening of unannotated genes

UCSC Genome Browser track that displays all analyzed transcription factor binding sites (TFBS) was performed to identify the transcription factors of the unannotated genes using DAVID database (Fujita et al., 2010), which was then visualized by constructing regulatory network using Cytoscape software (Kohl et al., 2011).

\section{Results}

\section{Intensity $Q C$ filtration}

The intensity QC of each sample was analyzed using Genotype Console software (Figure 1). The samples with contrast QC $<0.4$ (GSM811149_17T.CEL and GSM811154_14T.CEL) were excluded in the following research.

\section{Candidate genes with $\mathrm{LOH}$}

The candidate genes with $\mathrm{LOH}$ identified in this study were $\mathrm{MCU}$ (mitochondrial $\mathrm{Ca}^{2+}$ uniporter), $M I C U 1$ (mitochondrial calcium uniporter regulator 1) and OIT3 (oncoprotein induced transcript 3) on chromosome 10

Table 1. Partial results of LOH_SNP_gene

\begin{tabular}{|c|c|c|c|c|c|}
\hline Probe_id & $\mathrm{Chr}$ & Pos & CN_state & SNP_ID & Gene_ID \\
\hline SNP_A-8465056 & 10 & 74321221 & 2 & rs7921361 & $M I C U 1$ \\
\hline SNP_A-2002554 & 10 & 74334758 & 2 & rs6415911 & MICU1 \\
\hline SNP_A-8423526 & 10 & 74378437 & 2 & rs10823937 & MICU1 \\
\hline SNP_A-8716423 & 10 & 74417164 & 2 & rs9415071 & - \\
\hline SNP_A-1882206 & 10 & 74442817 & 2 & rs3009560 & - \\
\hline SNP_A-8631561 & 10 & 74490023 & 2 & rs 2921448 & $M C U$ \\
\hline SNP_A-8397978 & 10 & 74512727 & 2 & rs2894214 & $M C U$ \\
\hline SNP_A-2163625 & 10 & 74514615 & 2 & rs7086453 & $M C U$ \\
\hline SNP_A-2120808 & 10 & 74522284 & 2 & rs16930097 & $M C U$ \\
\hline SNP_A-2027945 & 10 & 74522494 & 2 & rs16930102 & $M C U$ \\
\hline SNP_A-2140867 & 10 & 74541864 & 2 & rs 11000413 & $M C U$ \\
\hline SNP_A-2297049 & 10 & 74570913 & 2 & rs7097134 & $M C U$ \\
\hline SNP_A-8486874 & 10 & 74606261 & 2 & rs12785228 & $M C U$ \\
\hline SNP_A-4259678 & 10 & 74672399 & 2 & rs 11000445 & OIT3 \\
\hline SNP_A-8465056 & 10 & 74321221 & 2 & rs7921361 & MICU1 \\
\hline SNP_A-2002554 & 10 & 74334758 & 2 & rs6415911 & $M I C U 1$ \\
\hline SNP_A-8465056 & 10 & 74321221 & 4 & rs7921361 & MICU1 \\
\hline SNP_A-2002554 & 10 & 74334758 & 4 & rs6415911 & MICU1 \\
\hline SNP_A-8423526 & 10 & 74378437 & 4 & rs10823937 & MICU1 \\
\hline SNP_A-8716423 & 10 & 74417164 & 4 & rs9415071 & - \\
\hline SNP_A-1882206 & 10 & 74442817 & 4 & rs3009560 & - \\
\hline SNP_A-8631561 & 10 & 74490023 & 4 & rs2921448 & $M C U$ \\
\hline SNP_A-8397978 & 10 & 74512727 & 4 & rs 2894214 & $M C U$ \\
\hline SNP_A-2163625 & 10 & 74514615 & 4 & rs7086453 & $M C U$ \\
\hline SNP_A-2120808 & 10 & 74522284 & 4 & rs16930097 & $M C U$ \\
\hline SNP_A-2027945 & 10 & 74522494 & 4 & rs16930102 & $M C U$ \\
\hline SNP_A-2140867 & 10 & 74541864 & 4 & rs 11000413 & $M C U$ \\
\hline SNP_A-2297049 & 10 & 74570913 & 4 & rs7097134 & $M C U$ \\
\hline SNP_A-8486874 & 10 & 74606261 & 4 & rs12785228 & $M C U$ \\
\hline SNP_A-4259678 & 10 & 74672399 & 4 & rs 11000445 & OIT3 \\
\hline
\end{tabular}

Chr, chromosome; Pos, the loci of SNP on chromosome; CN_state, the copy numbers; SNP_ID, the ID of SNP in dbSNP database; Gene_ID, the ID of gene corresponding to the SNP; LOH, loss of heterozygosity; SNP, single-nucleotide polymorphism 


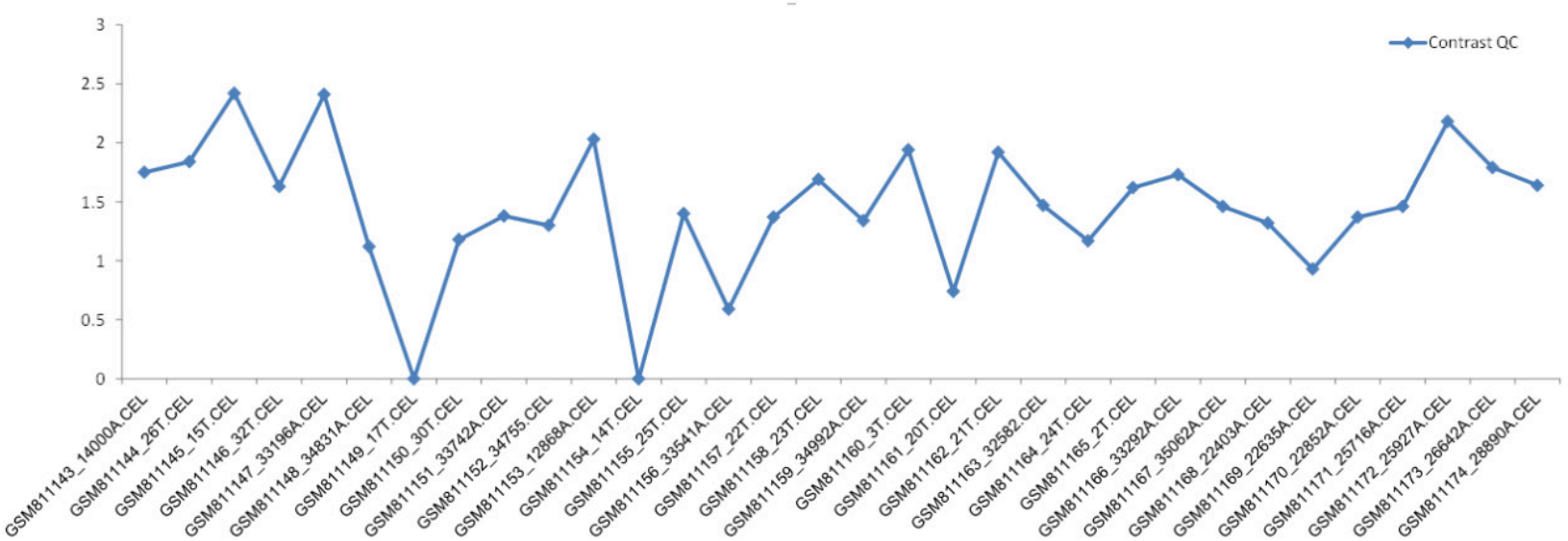

Figure 1. The Intensity QC in Each Sample. The X-axis represents file names of samples and the Y-axis represents the value of contrast QC. QC, quality control

Table 2. The Annotation Results of OIT3

\begin{tabular}{|c|c|}
\hline ID & OIT3 \\
\hline Gene Name & oncoprotein induced transcript 3 \\
\hline GOTERM_CC_FAT & $\begin{array}{l}\text { GO:0005635 nuclear envelope,GO:0012505 endomembrane system,GO:0031967 organelle } \\
\text { envelope,GO:0031975 envelope }\end{array}$ \\
\hline GOTERM_MF_FAT & $\begin{array}{l}\text { GO:0005509 calcium ion binding,GO:0043167 ion binding,GO:0043169 cation } \\
\text { binding,GO:0046872 metal ion binding }\end{array}$ \\
\hline INTERPRO & $\begin{array}{l}\text { IPR000152:EGF-type aspartate/asparagine hydroxylation conserved site,IPR001507:Endoglin/CD105 } \\
\text { antigen,IPR001881:EGF-like calcium-binding,IPR006209:EGF,IPR006210:EGF-like,IPR013032:EGF- } \\
\text { like region, conserved site,IPR017976:Endoglin/CD105 antigen subgroup,IPR017977:Endoglin/ } \\
\text { CD105 antigen conserved } \\
\text { site, IPR018097:EGF-like calcium-binding, conserved site }\end{array}$ \\
\hline PFAM & PF00008:227-262,PF00008:EGF,PF00100:Zona pellucida-like domain,PF00100:Zona_pellucida \\
\hline SMART & SM00179:EGF_CA,SM00181:EGF,SM00241:ZP \\
\hline
\end{tabular}

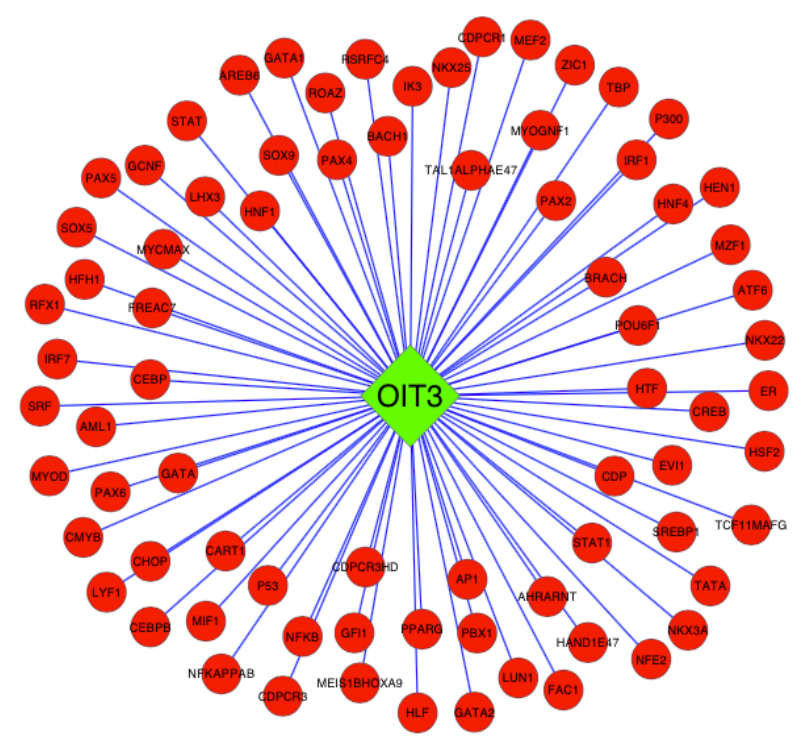

Figure 2. The Regulatory Network Between OIT3 and its Transcription Factors. The rhombus represents OIT3 and the surrounding circles represent transcription factors

(Partial results were shown in Table 1). The copy number (CN) in the samples were two or four in this study, probably suggesting LOH resulted from heterozygous deletion of one of the two alleles, or duplication of a maternal or paternal chromosome or chromosomal region and concurrent loss of the other allele.

\section{Gene annotation}

Gene annotation was performed to identify the functions of candidate genes with $\mathrm{LOH}$ using Gene database in NCBI. Accordingly, $M C U$ (Gene ID: 90550) was found to encode a calcium transporter that localizes to the mitochondrial inner membrane and interacts with mitochondrial calcium uptake (Bick et al., 2012; Csordas et al., 2012; Raffaello et al., 2012; Curry et al., 2013; Patron et al., 2013). MICU1 (Gene ID: 10367) could encode an essential regulator of mitochondrial $\mathrm{Ca}^{2+}$ uptake under basal conditions. The encoded protein interacts with the mitochondrial calcium uniporter and is essential in preventing mitochondrial $\mathrm{Ca}^{2+}$ overload that could cause excessive production of reactive oxygen species and cell stress (Mallilankaraman et al., 2012; Arvizo et al., 2013; Chen et al., 2013; Hoffman et al., 2013; Logan et al., 2014). OIT3 didn't obtain any annotated results from Gene database, but get some other annotated messages from GO, INTERPRO, PFAM, SMART annotation analysis provided by DAVID (Table 2). OIT3, oncoprotein induced transcript 3 , was correlated with calcium ion binding, ion binding and cation binding.

\section{Transcription factors of OIT3}

By performing UCSC TFBS analysis, this study identified a large number of transcription factors including STAT (signal transducer and activator of transcription), SOX9 (sex determining region Y-box 9), CREB (cAMP responsive element binding protein), $N F-k B$ (nuclear factor kappa B), PPARG (peroxisome proliferatoractivated receptor gamma) and $p 53$, which had regulatory effects on OIT3 (Figure 2). 


\section{Discussion}

By mapping the SNPs showing $\mathrm{LOH}$ in the tumor versus matched normal samples, this study identified three candidate genes with $\mathrm{LOH}$ (MICU1, MCU and OIT3) on chromosome 10 in pancreatic cancer, implying an important role for these genes in pancreatic cancer.

$M C U$ and MICU1 are two genes that have been demonstrated to play important roles in mitochondrial $\mathrm{Ca}^{2+}$ uptake (Perocchi et al., 2010; Baughman et al., 2011; Csordas et al., 2013). The $\mathrm{Ca}^{2+}$ handling by mitochondria is involved in cell life by triggering or preventing apoptosis probably functioning through the released pro-apoptotic factors such as Bax and Bak from the intermembrane space (Scorrano et al., 2003; Kroemer et al., 2007; Contreras et al., 2010). Thus, it could be speculated that $M C U$ and MICU1 may be related to the cancer progression via affecting cellular apoptosis. As expected, previous study has reported the down-regulation of $M C U$ targeted by cancer-related microRNA may increase cancer cell survival and contribute to tumorigenesis in various cancer cells (Marchi et al., 2013). Silencing MICU1 is also revealed to initiate the mitochondrial pathway for apoptosis by decreasing Bcl-2 expression together with increasing caspase- 3 activity and cytosolic cytochrome c contents (Arvizo et al., 2013). Therefore, $M C U$ and MICU1 may be oncogene or tumor suppressor gene and the LOH in them may lead to their lower expression, thus preventing or promoting pancreatic cancer cell apoptosis.

OIT3 located at 10q22.1 was another gene with $\mathrm{LOH}$ found in pancreatic cancer. This gene, also termed as liver-specific zona pellucida domain-containing protein (LZP), is related to hepatocellular function and could be used as a potential diagnostic biomarker for hepatocellular carcinoma (Xu et al., 2003). Also, OIT3 is reported to experience copy number losses and down-regulation in colorectal cancer (Yoshida et al., 2010). However, the details of the relationships between OIT3 and cancers are not clear. Herein, OIT3 was found to be associated with calcium ion binding, probably implying an involvement of this gene in cellular $\mathrm{Ca}^{2+}$ homeostasis. Moreover, based on the regulatory network in this study, OIT3 was regulated by a large number of transcription factors including STAT, SOX9, CREB, NF-kB, PPARG and p53 which were previously reported to be associated with pancreatic cancer. STAT-3, PPARG and NF-kB signaling pathways are involved in apoptosis and cellular differentiation in pancreatic cancer cells (Elnemr et al., 2000; Sahu and Srivastava, 2009). CREB is related to activate STAT-3 and cyclin D1 expression in pancreatic cancer cells which lead to cell proliferation and tumor progression (Zhang et al., 2010). SOX9 could initiate and accelerate the formation of premalignant lesions of pancreatic cancer (Kopp et al., 2012). In addition, mutations in p53 tumor suppressor gene are considered to drive metastasis and contribute to the carcinogenesis of pancreatic cancer (Sato et al., 1996; Amaya et al., 2004; Morton et al., 2010). The LOH of OIT3 may suggest dysregulated functions of these transcription factors, which may contribute to the progression of pancreatic cancer.

In conclusion, our global genomic analysis of SNPs provides evidence of LOH in MICU1, MCU and OIT3 on chromosome 10 in pancreatic cancer. They may be involved in pancreatic cancer progression by regulating the calcium ion homogenizes and cell apoptosis. However, future mechanistic researches will be required to determine the molecular mechanisms.

\section{Acknowledgements}

We wish to express our warm thanks to Fenghe(Shanghai) Information Technology Co., Ltd. Their ideas and help gave a valuable added dimension to our research.

\section{References}

Amaya K, Ohta T, Kitagawa H, et al (2004). Angiotensin II activates MAP kinase and $N F-k B$ through angiotensin II type I receptor in human pancreatic cancer cells. Int J Oncol, 25, 849-56.

Arvizo RR, Moyano DF, Saha S, et al (2013). Probing novel roles of the mitochondrial uniporter in ovarian cancer cells using nanoparticles. J Biol Chem, 288, 17610-8.

Baker DJ, Jin F, Jeganathan KB, et al (2009). Whole chromosome instability caused by Bub1 insufficiency drives tumorigenesis through tumor suppressor gene loss of heterozygosity. Cancer cell, 16, 475-86.

Baughman JM, Perocchi F, Girgis HS, et al (2011). Integrative genomics identifies $M C U$ as an essential component of the mitochondrial calcium uniporter. Nature, 476, 341-5.

Beroukhim R, Lin M, Park Y, et al (2006). Inferring loss-ofheterozygosity from unpaired tumors using high-density oligonucleotide SNP arrays. PLoS Comput Biol, $2,41$.

Bick AG, Calvo SE, Mootha VK (2012). Evolutionary diversity of the mitochondrial calcium uniporter. Science, 336, 886-.

Butz J, Wickstrom E, Edwards J (2003). Characterization of mutations and loss of heterozygosity of $p 53$ and K-ras 2 in pancreatic cancer cell lines by immobilized polymerase chain reaction. BMC Biotechnol, $\mathbf{3}, 11$.

Chen K, Hsu L-T, Wu C-Y, et al (2013). CBARA1 plays a role in stemness and proliferation of human embryonic stem cells. PloS One, 8, 63653.

Contreras L, Drago I, Zampese E, et al (2010). Mitochondria: the calcium connection. BBA-Gen Subjects, 1797, 607-18.

Csordas G, Golenar T, Seifert EL, et al (2013). MICU1 controls both the threshold and cooperative activation of the mitochondrial $\mathrm{Ca}^{2+}$ uniporter. Cell Metab, 17, 976-87.

Csordas G, Varnai P, Golenar T, et al (2012). Calcium transport across the inner mitochondrial membrane: molecular mechanisms and pharmacology. Mol Cell Endocrinol, 353, 109-13.

Curry MC, Peters AA, Kenny PA, et al (2013). Mitochondrial calcium uniporter silencing potentiates caspase-independent cell death in MDA-MB-231 breast cancer cells. Biochem Biophys Res Commun, 434, 695-700.

Day IN (2010). dbSNP in the detail and copy number complexities. Hum Mutat, 31, 2-4.

Donahue TR, Tran LM, Hill R, et al (2012). Integrative survivalbased molecular profiling of human pancreatic cancer. Clin Cancer Res, 18, 1352-63.

Elnemr A, Ohta T, Iwata K, et al (2000). PPARgamma ligand (thiazolidinedione) induces growth arrest and differentiation markers of human pancreatic cancer cells. Int J Oncol, 17, 1157-221.

Frampton SJ, King EV (2013). Loss of heterozygosity. in 'encyclopedia of otolaryngology, head and neck surgery', 


\section{Eds Springer, 1488}

Franko J, Krasinskas AM, Nikiforova MN, et al (2008). Loss of heterozygosity predicts poor survival after resection of pancreatic adenocarcinoma. J Gastrointest Surg, 12, 1664-73.

Fujita PA, Rhead B, Zweig AS, et al (2010). The UCSC genome browser database: update 2011. Nucleic Acids Res, 963.

Hoffman NE, Chandramoorthy HC, Shamugapriya S, et al (2013). MICU1 motifs define mitochondrial calcium uniporter binding and activity. Plant Cell Rep, 5, 1576-88.

Kohl M, Wiese S, Warscheid B (2011). Cytoscape: software for visualization and analysis of biological networks., Eds Springer, 696, 291-303

Kopp JL, von Figura G, Mayes E, et al (2012). Identification of Sox9-dependent acinar-to-ductal reprogramming as the principal mechanism for initiation of pancreatic ductal adenocarcinoma. Cancer Cell, 22, 737-50.

Kroemer G, Galluzzi L, Brenner C (2007). Mitochondrial membrane permeabilization in cell death. Physiol Rev, 87, 99-163.

Lin L-J, Asaoka Y, Tada M, et al (2008). Integrated analysis of copy number alterations and loss of heterozygosity in human pancreatic cancer using a high-resolution, single nucleotide polymorphism array. Oncol, 75, 102-12.

Logan CV, Szabadkai G, Sharpe JA, et al (2014). Loss-offunction mutations in MICU1 cause a brain and muscle disorder linked to primary alterations in mitochondrial calcium signaling. Nat Genet, 46, 188-93.

Lucas AL, Shakya R, Lipsyc MD, et al (2013). High prevalence of $B R C A 1$ and $B R C A 2$ germline mutations with loss of heterozygosity in a series of resected pancreatic adenocarcinoma and other neoplastic lesions. Clin Cancer Res, 19, 3396-403.

Mallilankaraman K, Doonan P, Cardenas C, et al (2012). MICU1 Is an essential gatekeeper for $M C U$-mediated mitochondrial $\mathrm{Ca}<\sup >2+</$ sup $>$ uptake that regulates cell survival. Cell, 151, 630-44.

Marchi S, Lupini L, Patergnani S, et al (2013). Downregulation of the mitochondrial calcium uniporter by cancer-related miR-25. Curr Biol, 23, 58-63.

Morton JP, Timpson P, Karim SA, et al (2010). Mutant p53 drives metastasis and overcomes growth arrest/senescence in pancreatic cancer. Proc Natl Acad Sci, 107, 246-51.

Patron M, Raffaello A, Granatiero V, et al (2013). The mitochondrial calcium uniporter $(M C U)$ : molecular identity and physiological roles. J Biol Chem, 288, 10750-8.

Perocchi F, Gohil VM, Girgis HS, et al (2010). MICU1 encodes a mitochondrial EF hand protein required for $\mathrm{Ca}^{2+}$ uptake. Nature, 467, 291-6.

Raffaello A, De Stefani D, Rizzuto R (2012). The mitochondrial $\mathrm{Ca}^{2+}$ uniporter. Cell Calcium, 52, 16-21.

Sahu RP, Srivastava SK (2009). The role of STAT-3 in the induction of apoptosis in pancreatic cancer cells by benzyl isothiocyanate. J Natl Cancer Inst, 101, 176-93.

Sato Y, Nio Y, Song M, et al (1996). p53 protein expression as prognostic factor in human pancreatic cancer. Anticancer Res, 17, 2779-88.

Scorrano L, Oakes SA, Opferman JT, et al (2003). BAX and BAK regulation of endoplasmic reticulum $\mathrm{Ca}^{2+}$ : a control point for apoptosis. Science, 300, 135-9.

Sherman BT, Huang dW, Tan Q, et al (2007). DAVID Knowledgebase: a gene-centered database integrating heterogeneous gene annotation resources to facilitate highthroughput gene functional analysis. BMC Bioinformatics, $8,426$.

Staaf J, Lindgren D, Vallon-Christersson J, et al (2008). Segmentation-based detection of allelic imbalance and loss- of-heterozygosity in cancer cells using whole genome SNP arrays. Genome Biol, 9, 136.

Xu ZG, Du JJ, Zhang X, et al (2003). A novel liver-specific zona pellucida domain containing protein that is expressed rarely in hepatocellular carcinoma. Hepatology, 38, 735-44.

Yoshida T, Kobayashi T, Itoda M, et al (2010). Clinical omics analysis of colorectal cancer incorporating copy number aberrations and gene expression data. Cancer Informatics, 9, 147.

Zhang Y, Bharadwaj U, Logsdon CD, et al (2010). ZIP4 regulates pancreatic cancer cell growth by activating IL-6/STAT3 pathway through zinc finger transcription factor $C R E B$. Clin Cancer Res, 16, 1423-30. 